\title{
Preferred reporting items for systematic review and meta-analysis protocols (PRISMA-P) 2015: elaboration and explanation
}

This Research Methods and Reporting paper (BMJ

2015;350:g7647, doi:10.1136/bmj.g7647) should have the volume number 350 (not 349). 